\title{
HLA-A*24:02 as a common risk factor for antiepileptic drug-induced cutaneous adverse reactions OPEN
}

Yi-Wu Shi, PhD* Fu-Li Min, MD* Dong Zhou, MD Bin Qin, MD Juan Wang, MS Fa-Yun $\mathrm{Hu}, \mathrm{MD}$ Ying-Kit Cheung, PhD Jin-Hua Zhou, MD Xiang-Shu $\mathrm{Hu}, \mathrm{MD}$ Jue-Qian Zhou, MD Lie-Min Zhou, MD Zhong-zheng Zheng, MS Jie Pan, MS

$\mathrm{Na} \mathrm{He}, \mathrm{MD}$ Zhi-Sheng Liu, MD Yun-Qi Hou, MS Kheng-Seang Lim, MD Yang-mei Ou, MS Amy Hui-Ping Khor, MS Ching-Ching $\mathrm{Ng}$, PhD Bi-Jun Mao, MS Xiao-Rong Liu, MD Bing-Mei Li, MD Yao-Yun Kuan, MS Yong-Hong Yi, MD, PhD Xue-lian He, MS

Xiao-Yan Deng, MS Tao Su, PhD Patrick Kwan, MD, PhD Wei-Ping Liao, MD, PhD

Correspondence to Dr. Liao: wpliao@163.net or Dr. Kwan: patrick.kwan@unimelb.edu.au

\section{Supplemental data} at Neurology.org

\section{ABSTRACT}

Objective: To investigate the involvement of human leukocyte antigen (HLA) loci in aromatic antiepileptic drug-induced cutaneous adverse reactions.

Methods: A case-control study was performed to detect HLA loci involved in aromatic antiepileptic drug-induced Stevens-Johnson syndrome in a southern Han Chinese population. Between January 1, 2006, and December 31, 2015, 91 cases of Stevens-Johnson syndrome induced by aromatic antiepileptic drugs and 322 matched drug-tolerant controls were enrolled from 8 centers. Important genotypes were replicated in cases with maculopapular eruption and in the meta-analyses of data from other populations. Sequence-based typing determined the HLA-A, HLA-B, HLA-C, and HLA-DRB1 genotypes.

Results: HLA-B*15:02 was confirmed as strongly associated with carbamazepine-induced Stevens-Johnson syndrome $\left(p=5.63 \times 10^{-15}\right)$. In addition, HLA-A*24:02 was associated significantly with Stevens-Johnson syndrome induced by the aromatic antiepileptic drugs as a group $\left(p=1.02 \times 10^{-5}\right)$ and by individual drugs (carbamazepine $p=0.015$, lamotrigine $p=0.005$, phenytoin $p=0.027)$. Logistic regression analysis revealed a multiplicative interaction between HLA-B*15:02 and HLA-A*24:02. Positivity for HLA-A*24:02 and/or HLA-B*15:02 showed a sensitivity of $72.5 \%$ and a specificity of $69.0 \%$. The presence of HLA-A*24:02 in cases with maculopapular exanthema was also significantly higher than in controls ( $p=0.023$ ). Metaanalysis of data from Japan, Korea, Malaysia, Mexico, Norway, and China revealed a similar association.

Conclusions: HLA-A*24:02 is a common genetic risk factor for cutaneous adverse reactions induced by aromatic antiepileptic drugs in the southern Han Chinese and possibly other ethnic populations. Pretreatment screening is recommended for people in southern China. Neurology ${ }^{\circledR}$ 2017;88:2183-2191

\section{GLOSSARY}

AED = antiepileptic drug; $\mathbf{C A D R}=$ cutaneous adverse drug reaction; $\mathbf{C B Z}=$ carbamazepine; $\mathbf{H L A}=$ human leukocyte antigen; LTG = lamotrigine; MPE = maculopapular exanthema; PHT = phenytoin; SJS $=$ Stevens-Johnson syndrome; TEN = toxic epidermal necrolysis.

Cutaneous adverse drug reactions (cADRs) include mild maculopapular exanthema (MPE) and severe cutaneous reactions such as hypersensitivity syndrome, Stevens-Johnson syndrome (SJS), and toxic epidermal necrolysis (TEN). ${ }^{1}$ cADRs are considered a major public health issue because of their potentially life-threatening morbidity, especially severe cutaneous reactions. The incidence of SJS/TEN is estimated to vary from 1 in 1,000 to 10,000 drug exposures, ${ }^{2,3}$ and its mortality is as high as 35\%.4,5 Many drugs are associated with cADRs; however, the majority of severe cutaneous reactions cases are caused by a few drug classes. ${ }^{6}$ Antiepileptic drugs (AEDs), particularly those with aromatic ring structures such as carbamazepine (CBZ), lamotrigine (LTG), and phenytoin (PHT), are among the most common

\footnotetext{
*These authors contributed equally to this work.

Author affiliations are provided at the end of the article.

Go to Neurology.org for full disclosures. Funding information and disclosures deemed relevant by the authors, if any, are provided at the end of the article. The Article Processing Charge was funded by Science and Technology Project of Guangzhou.

This is an open access article distributed under the terms of the Creative Commons Attribution-NonCommercial-NoDerivatives License 4.0 (CC BY-NC-ND), which permits downloading and sharing the work provided it is properly cited. The work cannot be changed in any way or used commercially without permission from the journal.
} 
causes of severe cutaneous reactions. ${ }^{1,7}$ The incidence of AED-induced SJS was estimated as $0.2 \%$, and all cases occurred in individuals receiving aromatic AEDs. ${ }^{8}$

Several human leukocyte antigen (HLA) markers have been identified for druginduced cADRs. HLA-B*57:01 was associated with abacavir-induced hypersensitivity reaction. ${ }^{9,10}$ HLA-B*58:01 was associated with allopurinol-induced SJS/TEN. ${ }^{11}$ HLA$B^{*} 15: 02$ is a high-risk genetic marker for CBZ-induced SJS/TEN in Han Chinese individuals living in Taiwan. ${ }^{12}$ This finding was confirmed in southern Han Chinese ${ }^{13,14}$ and populations in southeast $\mathrm{Asia}^{15-17}$ and has resulted in breakthroughs in the prevention of CBZ-induced SJS/TEN. ${ }^{18}$ Subsequently, cases of CBZ-induced SJS/TEN that were negative for HLA-B*15:02 in Chinese, ${ }^{19,20}$ Indian, Thai, and Malay populations were reported. ${ }^{15-17}$ In our extended study, we revealed a potential association between HLA-A*24:02 and CBZ-induced SJS/TEN in a southern Han Chinese population. ${ }^{14,21}$ These findings suggested that in addition to HLA-B*15:02, other genetic factors might contribute to CBZ-induced SJS/ TEN. The relationships between HLA genes and the other commonly used aromatic AEDs, including LTG and PHT, are unclear.

To examine the HLA loci involved in aromatic AED-induced SJS, we conducted a multicenter case-control study in southern China. Significant findings were replicated in MPE cases and validated with meta-analyses of data from independent studies in other populations.

METHODS Recruitment of cases and drug-tolerant controls. Cases included individuals who developed cADRs after receiving aromatic AEDs. The Roujeau diagnostic criteria were used to diagnose and classify cADRs, ${ }^{5,22}$ and a dermatologist confirmed the diagnoses on the basis of the clinical morphology of the skin damage. SJS is defined as mucocutaneous disorders characterized by extensive erythema, blisters, epidermal detachment, enanthema, and skin detachment of $<10 \%$ of the body surface area. TEN is defined as skin detachment $>30 \%$. The SJS-TEN overlap is defined as skin detachment of $10 \%$ to $30 \%$. MPE is characterized by cutaneous fine pink macules and papules, and lesions without mucosal or systemic involvement. Cases were defined as participants who developed cADRs within 8 weeks of beginning an aromatic AED for which no other causes were found. No cases were taking other medicines associated with SJS/ TEN such as abacavir and allopurinol.

We recruited cases between 2006 and 2015 from 8 hospitals in 4 regions in southern China, including Guangdong, Hong
Kong, Hubei, and Sichuan. As a part of the follow-up after AEDs were prescribed, individuals were asked to return to the hospital if they developed cADRs. Controls were participants with epilepsy who took aromatic AEDs for at least 3 months without evidence of cutaneous adverse reactions. To determine whether the association between HLA and cADRs was drug-specific and ethnicityspecific, controls were Han Chinese from the same region who took the same AEDs as the matched cases. A matching ratio of at least 3:1 (controls:cases) was used.

To replicate any significant findings, a cohort of MPE cases was recruited separately from 2007 to 2015 in Guangzhou, Guangdong province.

All individuals enrolled were unrelated ethnic Han Chinese. None of the biological grandparents of the participants were from other races.

HLA high-resolution genotyping. We extracted genomic DNA from peripheral blood using the QIARamp blood minikit (Qiagen, Hilden, Germany). The Shanghai Tissuebank Biotechnology (Shanghai, China) performed the high-resolution sequence-based HLA typing. An ABI 3730 sequencer (Applied Biosystems, Foster City, CA) was used to perform the sequencing for HLA genotyping. The primers used for PCR amplification of DNA fragments of the HLA-A, HLA-B, HLA-C, and HLADRB1 genes were as follows: (1) AF and AR for HLA-A alleles, (2) BF and BR for HLA-B alleles, (3) CF and CR for HLA-C alleles, and (4) DRB1F and DRB1R for HLA-DRB1 alleles (table e-1 at Neurology.org). The primers used for resequencing of the HLA-A, HLA-B, HLA-C, and HLA-DRB1 genes are also listed in table e-1. Sequence alignment was used to determine the 4-digit HLA alleles with the software vector NTI 6.0 (Informax Inc, Gaithersburg, MD).

Meta-analysis. To validate the findings from the present cohort, we performed meta-analyses on data obtained from other populations. A complete search of online databases, including MEDLINE, EMBASE, Google Scholar, the Chinese National Knowledge Infrastructure, and the China Science and Technology Journal, were conducted. Detailed information regarding the search method and criteria of selection and exclusion is presented in the e-supplement. Data from 14 studies were used for the meta-analyses. These studies included populations from Japan, Korea, Malaysia, Mexico, Norway, and China.

Standard protocol approvals, registrations, and patient consents. The studies adhered to the guidelines of the International Committee of Medical Journal Editors with regard to patient consent for research or participation and received approval from local ethics committees of the participating hospitals.

The Ethics Committee of the Second Affiliated Hospital of Guangzhou Medical University provided ethics approval.

Statistical analysis. SPSS version 19.0 (SPSS Inc, Chicago, IL) was used to perform the statistical analyses. Two-by-two $\chi^{2}$ tests were performed to compare the allele carrier rates between groups. Logistic regression was used to analyze the possible interaction between HLA-A*24:02 and HLA-B*15:02.Values of $p<0.05$ (2-sided) were considered statistically significant. Bonferroni correction for $p$ values was applied when multiple comparisons were performed (e-supplement).

RESULTS HLA and SJS/TEN. Overall findings. Ninetyone cases with aromatic AED-induced SJS/TEN (56 CBZ-SJS/TEN, 22 LTG-SJS, and 13 PHT-SJS) and 322 controls (180 exposed to CBZ, 102 to LTG, and 40 to PHT) were included. Table e- 2 summarizes the 
demographic variables of the cases and controls. There were no significant differences between the case and control groups in mean age, sex ratio, and the presence of major comorbidities. As expected, the CBZ and LTG controls showed significantly higher exposure doses than the cases. Similarly, the controls showed a higher rate of concomitant aromatic AED use. Most cases received aromatic AEDs to treat epilepsy, except for 9 patients who received AEDs to treat neuropathies (6 cases), psychiatric illness ( 1 case), blepharospasm (1 case), and postoperation prophylaxis ( 1 case). For the 9 individuals with nonepileptic indications, we failed to find matching controls with the same indications. Because of the possible risk of bias, 2 situations, i.e., with or without cases without epilepsy, were considered separately in the statistical analysis. There was no difference in the results of the statistical analysis between the 2 scenarios.
The genotypes of HLA-A, HLA-B, HLA-C, and HLA-DRB1 in the 91 cases with CBZ-, LTG-, and PHT-induced SJS/TEN are shown in table e-3. HLA alleles that are associated significantly with cADRs from the 2-by-2 $\chi^{2}$ analyses are listed in table 1 , while comparisons of all alleles are shown in table e-4.

As shown in table 1, 6 alleles were detected as significant in the CBZ-induced SJS/TEN group: HLA-B*15:02, HLA-C*08:01, HLADRB1*12:02, HLA-A*24:02, HLA-B*15:11, and HLA-DRB $1 * 01: 01$. The HLA-C*08:01 and HLA-DRB $1 * 12: 02$ alleles are recognized as haplotype alleles of HLA-B*15:02, ${ }^{23}$ appearing with HLA-B* $15: 02$ in $90.6 \%$ (48 of 53 ) and $81.6 \%$ (31 of 38 ) of cases, respectively (table e-5). HLA-B*15:11 and HLA-DRB ${ }^{*} 01: 01$ appeared with HLA$B^{*} 15: 02$ and/or HLA-A*24:02 in all but 1 case each (table e-6). Thus, HLA-B*15:02 and HLA-A*24:02

Table 1 Risk factors for CBZ-, LTG-, and PHT-induced SJS/TEN

\begin{tabular}{|c|c|c|c|c|}
\hline \multirow[b]{2}{*}{ Allele } & \multicolumn{2}{|c|}{ HLA genotypes/total, n/N (\%) } & \multicolumn{2}{|l|}{ Cases vs controls } \\
\hline & SJS/TEN ${ }^{a}$ & Controls $^{a}$ & p Value & OR (95\% Cl) \\
\hline \multicolumn{5}{|l|}{ CBZ } \\
\hline$B * 15: 02$ & $39 / 56(69.64)$ & 28/179 (15.64) & $5.63 \times 10^{-15 b}$ & $12.37(6.16-24.86)$ \\
\hline$C * 08: 01^{c}$ & $42 / 55(76.36)$ & $38 / 177(21.47)$ & $7.36 \times 10^{-14}$ & $11.82(5.76-24.23)$ \\
\hline DRB1 $* 12: 02^{c}$ & 29/54 (53.7) & $45 / 176(25.57)$ & $1.08 \times 10^{-4}$ & 3.38 (1.79-6.36) \\
\hline$A * 24: 02$ & 17/56 (30.36) & 28/178 (15.73) & 0.015 & $2.33(1.16-4.69)$ \\
\hline$B * 15: 11^{d}$ & $4 / 56(7.14)$ & $0 / 179(0)$ & 0.003 & $30.77(1.63-580.87)$ \\
\hline DRB1*01:01 ${ }^{d}$ & $4 / 54(7.41)$ & $1 / 176(0.57)$ & 0.013 & 14.0 (1.53-128.10) \\
\hline \multicolumn{5}{|l|}{ LTG } \\
\hline$A * 24: 02$ & $10 / 22(45.45)$ & 16/102 (15.69) & $0.005^{b}$ & $4.48(1.66-12.11)$ \\
\hline \multicolumn{5}{|l|}{ PHT } \\
\hline$A * 24: 02$ & 6/13 (46.2) & $5 / 40(12.5)$ & 0.027 & $6.00(1.42-25.27)$ \\
\hline$A * 02: 01^{d}$ & 3/13 (23.1) & $1 / 40(2.5)$ & 0.042 & $11.7(1.10-124.84)$ \\
\hline \multicolumn{5}{|l|}{ Pooled } \\
\hline$B * 15: 02$ & $50 / 91$ (54.95) & $56 / 321(17.45)$ & $5.07 \times 10^{-13 b, e}$ & 5.77 (3.49-9.55) \\
\hline$C * 08: 01^{c}$ & $53 / 88(60.23)$ & 73/319 (22.88) & $1.97 \times 10^{-11}$ & 5.10 (3.09-8.42) \\
\hline DRB1*12:02c & $38 / 87$ (43.68) & $81 / 317$ (25.55) & 0.001 & 2.26 (1.38-3.70) \\
\hline$A * 24: 02$ & 33/91 (36.26) & 49/320 (15.31) & $1.02 \times 10^{-5 b, e}$ & 3.15 (1.86-5.32) \\
\hline$B * 15: 11^{d}$ & $4 / 91(4.40)$ & 2/321 (0.62) & 0.031 & 7.33 (1.32-40.7) \\
\hline$B * 15: 01$ & 8/91 (8.79) & $9 / 321(2.80)$ & 0.025 & 3.34 (1.25-8.93) \\
\hline
\end{tabular}

Abbreviations: $\mathrm{CBZ}$ = carbamazepine; $\mathrm{Cl}=$ confidence interval; $\mathrm{HLA}=$ human leukocyte antigen; $\mathrm{LTG}=$ lamotrigine; $\mathrm{OR}=$ odds ratio; PHT = phenytoin; SJS/TEN = Stevens-Johnson syndrome/toxic epidermal necrolysis.

${ }^{a}$ Several individuals were not subjected to HLA genotyping because of insufficient DNA.

${ }^{b}$ Significant after Bonferroni correction (e-supplement and table e-4), $p=1.35 \times 10^{-13}, \mathrm{n}=24$ for HLA-B*15:02 correction in CBZ-SJS; $p=0.04, n=8$ for HLA-A*24:02 correction in LTG-SJS; $p=1.93 \times 10^{-11}, \mathrm{n}=38$ for HLA$B * 15: 02$ correction in SJS pooled data; and $p=1.43 \times 10^{-4}, n=14$ for HLA-A*24:02 correction in SJS pooled data. ${ }^{c}$ The haplotype allele of HLA-B*15:02.

${ }^{d}$ These alleles appeared with HLA-B*15:02 or HLA-A*24:02 in all but 1 or 2 cases each.

e When the 9 cases without epilepsy were excluded, $p=6.74 \times 10^{-13}$, OR 6.05 (95\% CI 3.59-10.20) for HLA-B*15:02; and $p=5.33 \times 10^{-6}$, OR $3.36(95 \% \mathrm{Cl} 1.96-5.77)$ for HLA-A*24:02. $p=2.56 \times 10^{-11}$ for HLA-B*15:02 and $p=7.46 \times$ $10^{-5}$ for HLA-A*24:02 after Bonferroni correction. 
remained the only significantly associated alleles. HLA-A*24:02 was a significantly associated allele in patients who were negative for HLA-B*15:02 (7 of 17 vs 24 of 149 in CBZ controls, odds ratio 3.65 , $95 \%$ confidence interval $1.26-10.52, p=0.029$ ). HLA-B*15:02/HLA-A*24:02 is not a common haplotype in southern Han Chinese (http://www. allelefrequencies.net). ${ }^{23,24}$ These data suggested that the HLA-A*24:02 allele is independently and significantly associated with CBZ-induced SJS/TEN.

In the LTG-induced SJS group, HLA-A*24:02 was the only significantly associated allele detected.

In the PHT-induced SJS group, in addition to HLA-A*24:02, HLA-A*02:01 was found to be marginally significantly associated but appeared alone in only 2 cases.

In the pooled analysis (table 1), HLA-B*15:02 and HLA-A*24:02 appeared to be associated alleles $\left(p=5.07 \times 10^{-13}\right.$ and $p=1.02 \times 10^{-5}$, respectively), and remained significant after Bonferroni correction $\left(p=1.93 \times 10^{-11}\right.$ and $p=1.43 \times$ $10^{-4}$, respectively, table e-2). In addition, HLA$B^{*} 15: 11$ and HLA-B*15:01 were potentially associated alleles.

HLA-B $\boldsymbol{B}^{*}$ 15:02. The carrier rate of HLA-B*15:02 was higher in the CBZ-induced SJS/TEN group compared with the control group $(p=5.63 \times$ $\left.10^{-15}\right)$. HLA-B*15:02 showed a sensitivity of $69.6 \%$ and specificity of $84.4 \%$ for CBZ-induced SJS/TEN. HLA-B*15:02 was present in $22.7 \%$ (5 of 22) of cases with LTG-induced SJS, 18.6\% (19 of 102) of LTG controls ( $p=0.89$ ), $46.2 \%$ (6 of 13) of cases with PHT-SJS, and 22.5\% (9 of 40) of PHT controls $(p=0.22)$. These findings suggested that HLA-B*15:02 is a high-risk but relatively specific factor for CBZ-induced SJS/TEN.
HLA-A*24:02. HLA-A*24:02 was present in 30.4\% (17 of 56) of patients with CBZ-induced SJS/TEN, which was more frequent than in CBZ controls (28 of $178,15.7 \%, p=0.015)$. In the LTG-induced SJS group, $45.4 \%$ (10 of 22) of cases carried HLA$A * 24: 02$, which was more frequent than in the LTG controls $(p=0.005, p=0.04$ after Bonferroni correction) (table e-2). Similarly, a significant difference was found for the presence of HLA-A*24:02 between cases with PHT-induced SJS and PHT controls (6/13 vs $5 / 40, p=0.027)$. These findings suggested that HLA-A*24:02 is a potential shared risk factor for CBZ-, LTG-, and PHT-induced SJS/TEN. The sensitivity and specificity were $30.4 \%$ and $84.3 \%$ for CBZ, $45.4 \%$ and $84.3 \%$ for LTG, and $46.2 \%$ and $87.5 \%$ for PHT, respectively.

Interaction between HLA-B and HLA-A. HLA-B* 15:02 and HLA-A*24:02 were present either alone or together in patients with AED-induced SJS/TEN (figure 1). Thus, we performed logistic regression analyses to examine the potential interaction between the 2 alleles (table 2). The odds ratios in SJS/TEN patients who were positive for both HLA-B*15:02 and HLA-A*24:02 were higher than in those patients who were positive for either HLA-B*15:02 or HLA$A * 24: 02$ alone. A potential multiplicative interaction between HLA-B*15:02 and HLA-A*24:02 was observed (odds ratio 1.31). The estimated excess risk from the interaction was 16.85. Combining HLA$A * 24: 02$ and HLA-B*15:02 into a single group (i.e., carriers of HLA-A*24:02 and/or HLA-B*15:02) increased the sensitivity to $82.1 \%$ for CBZ-SJS/TEN and $72.5 \%$ for the 3 AED-induced SJS/TENs (with specificities $70.6 \%$ and $69.0 \%$, respectively).

Additional logistic regression analyses were performed to examine the potential interactions between

Figure 1 Presence of HLA-B*15:02 and HLA-A*24:02 alleles in CBZ- (A), LTG- (B), and PHT- (C) induced SJS/TEN
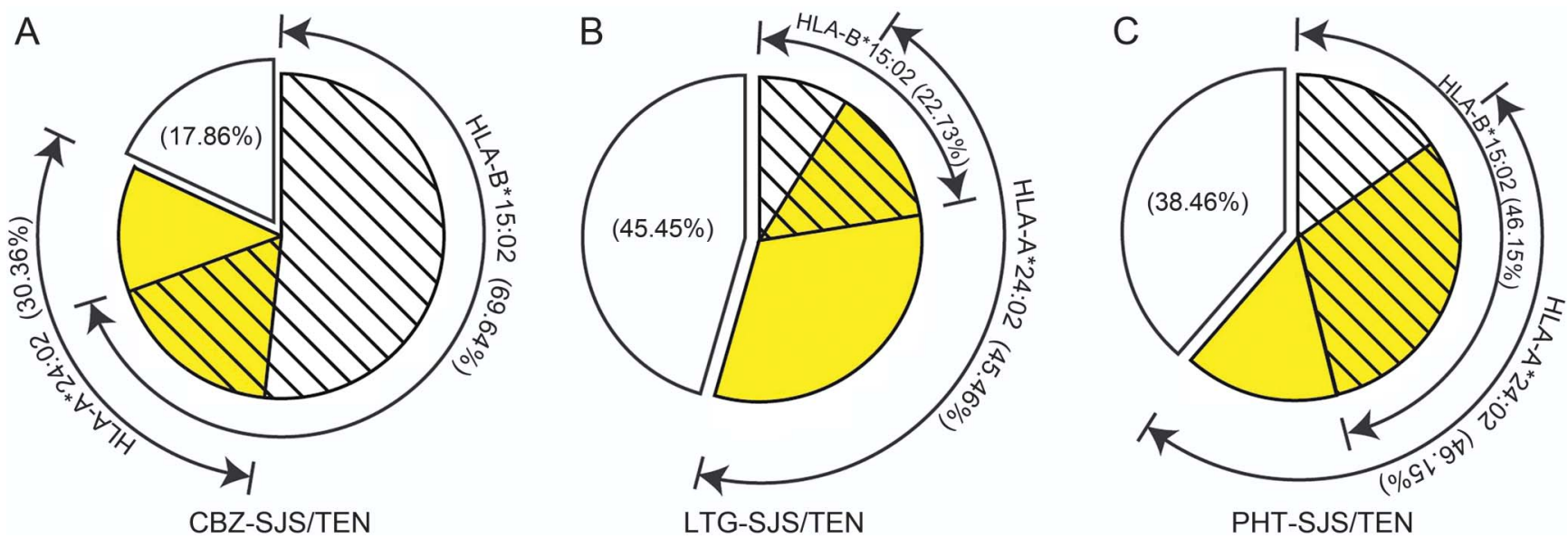

CBZ = carbamazepine; HLA = human leukocyte antigen; LTG = lamotrigine; PHT = phenytoin; SJS/TEN = Stevens-Johnson syndrome/toxic epidermal necrolysis. 


\begin{tabular}{|c|c|c|c|c|}
\hline Table 2 & $\begin{array}{l}\text { Excess risk from int } \\
\text { alleles in AED-induc }\end{array}$ & $\begin{array}{l}\text { n between } \\
\text { /TEN (logist }\end{array}$ & $\begin{array}{l}24: 02 \text { and } \\
\text { ession) }\end{array}$ & 15:02 \\
\hline HLA-A*24:02 & HLA-B*15:02 & SJS/TEN, $n$ & Controls, $n$ & OR \\
\hline+ & + & 17 & 6 & 24.93 \\
\hline+ & - & 16 & 43 & 3.27 \\
\hline- & + & 33 & 50 & 5.81 \\
\hline- & - & 25 & 220 & 1.0 \\
\hline
\end{tabular}

Abbreviations: AED = antiepileptic drug; HLA = human leukocyte antigen; OR = odds ratio; SJS/TEN = Stevens-Johnson syndrome/toxic epidermal necrolysis.

With the multiplicative model, the expected joint OR was 19.0 (3.27 $\times 5.81)$, and the OR of the multiplicative interaction was 1.31 (24.93/19.00). Under an additive model, the expected joint OR was $8.08(3.27+5.81-1)$, and the excess risk from interaction was $16.85(24.93-8.08)$

HLA-B*15:02 or HLA-A*24:02 and each of the other HLA alleles. Potential multiplicative interactions were found between HLA-A*24:02 and HLA$B * 15: 11$ (table e-7) and between HLA-B*15:02 and HLA-A*02:01 (table e-8).

HLA and MPE. Significant genotypes were replicated in 193 cases with MPE recruited in Guangzhou, including 134 with CBZ-induced MPE and 59 with LTG-induced MPE. HLA-A*24:02 was present in $24.2 \%$ ( 45 of 186) of cases with MPE, which was higher than in the controls $(15.7 \%, 44$ of $280, p=$ 0.023 ) (table 3 ). The frequency of HLA-B* $15: 02$ was similar between the 2 groups $(p=0.56)$.

Meta-analysis. To validate the association between HLA-A*24:02 and aromatic AED-induced cADRs, meta-analysis was performed using data from different populations (e-supplement and tables e-9 and e-10). Two types of data, i.e., from drug-tolerant controls and population controls, were analyzed separately. Compared with the tolerant controls, an

\begin{tabular}{|c|c|c|c|c|}
\hline Table 3 & $\begin{array}{l}\text { Association of HL } \\
\text { LTG-induced MPE }\end{array}$ & *24:02 and HLA & 1502 all & with CBZ- and \\
\hline \multirow[b]{2}{*}{ Allele } & \multicolumn{2}{|c|}{ HLA genotypes/total, n/N (\%) } & \multicolumn{2}{|c|}{ Cases vs controls } \\
\hline & $\mathrm{MPE}^{\mathrm{a}}$ & Controls $^{a}$ & $p$ Value & OR $(95 \% \mathrm{Cl})$ \\
\hline \multicolumn{5}{|l|}{ CBZ } \\
\hline$A * 24: 02$ & 32/131 (24.43) & 28/178 (15.73) & 0.056 & $1.73(0.98-3.05)$ \\
\hline$B * 15: 02$ & 19/132 (14.39) & 28/179 (15.64) & 0.761 & $0.91(0.48-1.71)$ \\
\hline \multicolumn{5}{|l|}{ LTG } \\
\hline$A * 24: 02$ & $13 / 55(23.64)$ & 16/102 (15.69) & 0.221 & $1.66(0.73-3.78)$ \\
\hline$B * 15: 02$ & 9/58 (15.52) & 19/102 (18.63) & 0.619 & $0.80(0.34-1.91)$ \\
\hline \multicolumn{5}{|l|}{ Pooled } \\
\hline$A * 24: 02$ & $45 / 186(24.19)$ & $44 / 280(15.71)$ & 0.023 & $1.71(1.08-2.73)$ \\
\hline B*15:02 & $28 / 190(14.74)$ & $47 / 281$ (16.73) & 0.563 & $0.86(0.52-1.43)$ \\
\hline
\end{tabular}

Abbreviations: $\mathrm{CBZ}$ = carbamazepine; $\mathrm{Cl}=$ confidence interval; $\mathrm{HLA}=$ human leukocyte antigen; $\mathrm{LTG}=$ lamotrigine; MPE = maculopapular exanthema; $\mathrm{OR}=$ odds ratio.

a Several individuals were not tested by HLA genotyping because of insufficient DNA. association between HLA-A*24:02 and MPE was found in data from Taiwan, Korea, Malaysia, and southern China $(p=0.046)$. There was also an association between HLA-A*24:02 and CADRs for data that did not specify SJS/TEN or MPE, based on data from Japan, Norway, and southern China $(p=$ 0.031) (figure 2A). Compared with the population controls, we found an overall association between HLA-A*24:02 and cADRs $(p=0.036)$ (figure 2B) in the data from Japanese, Korean, Mexican, and Chinese populations.

DISCUSSION CBZ, LTG, and PHT, which have similar chemical structures and contain an aromatic ring, are prescribed commonly to treat epilepsy, ${ }^{8,25}$ which is estimated to affect $\approx 1 \%$ of the world's population. ${ }^{26}$ However, these drugs are also among the most common causes of cADRs, especially SJS and TEN. ${ }^{8,25,27}$ CBZ-induced SJS/TEN is strongly associated with HLA-B*15:02 in southern Han Chinese $^{13,14}$ and populations in southeast Asia with varying sensitivity, ${ }^{15-17}$ and CBZ-SJS/TEN is associated with HLA-A*31:01 in whites and Japanese, with sensitivities of $42 \%$ and $60.7 \%$, respectively. ${ }^{28,29}$ However, HLA markers for LTG- and PHT-induced SJS/TEN have not been established firmly, although a Taiwanese study detected the CYP2C9*3 allele of the drug-metabolizing enzyme in a case of PHTinduced SJS/TEN. ${ }^{30}$ In the present study, HLA$A * 24: 02$ was revealed as a shared risk factor for SJS/ TEN induced by the aromatic AEDs CBZ, LTG, and PHT, while confirming that HLA-B*15:02 is a high risk factor for CBZ-induced SJS/TEN.

Early studies in Han Chinese living in Taiwan and Hong Kong showed that patients with CBZ-induced SJS/TEN were almost exclusively HLA-B*15:02 carriers. ${ }^{24,31}$ Cases of CBZ-induced SJS/TEN were reported in southern Han Chinese who were negative for HLA-B* $15: 02,{ }^{19}$ with a consistent negative rate of $\approx 30 \%$. HLA-A $* 24: 02$ was also noted as a potential risk factor. ${ }^{14,21}$ In the present study, $30.4 \%$ of patients with CBZ-induced SJS/TEN were negative for HLA-B*15:02, and HLA-A*24:02 was revealed as a risk factor for CBZ-induced SJS/TEN. The carrier rate of HLA-A*24:02 was higher in patients with CBZ-induced SJS/TEN who were negative for HLA$B * 15: 02$, suggesting that HLA-A*24:02 is an independent risk factor for CBZ-induced SJS/TEN. Furthermore, HLA-A*24:02 was associated with SJS/ TEN induced by LTG and PHT, suggesting that HLA-A*24:02 is a common risk factor for aromatic AED-induced SJS/TEN.

HLA-B*15:02 was associated with PHT- or LTGinduced SJS/TEN in Taiwan and Hong Kong Han Chinese. ${ }^{31-33} \mathrm{We}$ also found a relatively high carrier rate of HLA-B* 15:02 in PHT-induced SJS (46.16\%) 
A. Presence of the HLA-A $24: 02$ allele in AED-induced cADRs and tolerant controls

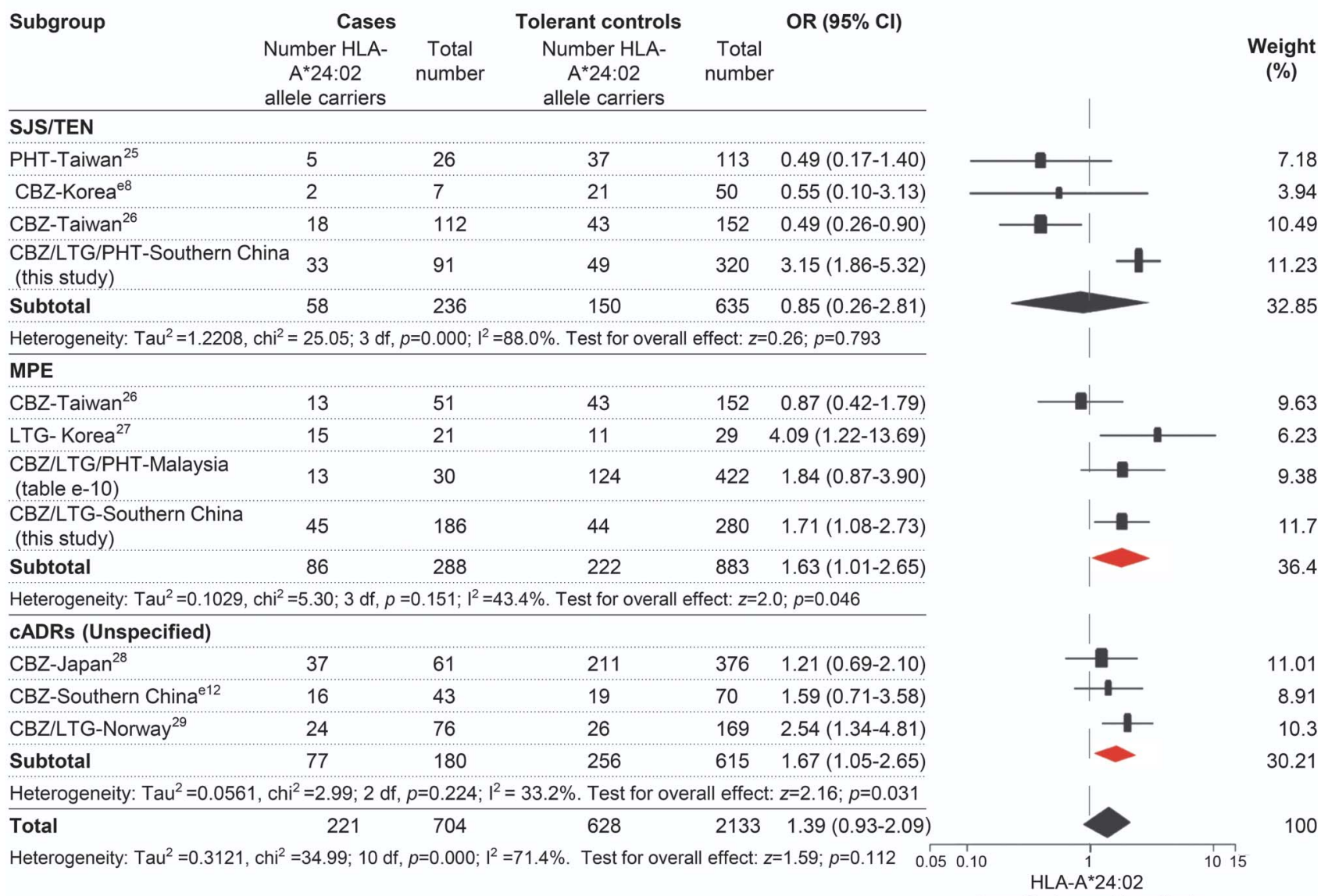

B. Frequency of the HLA-A*24:02 allele in AED-induced CADRs and population controls Less likely More likely

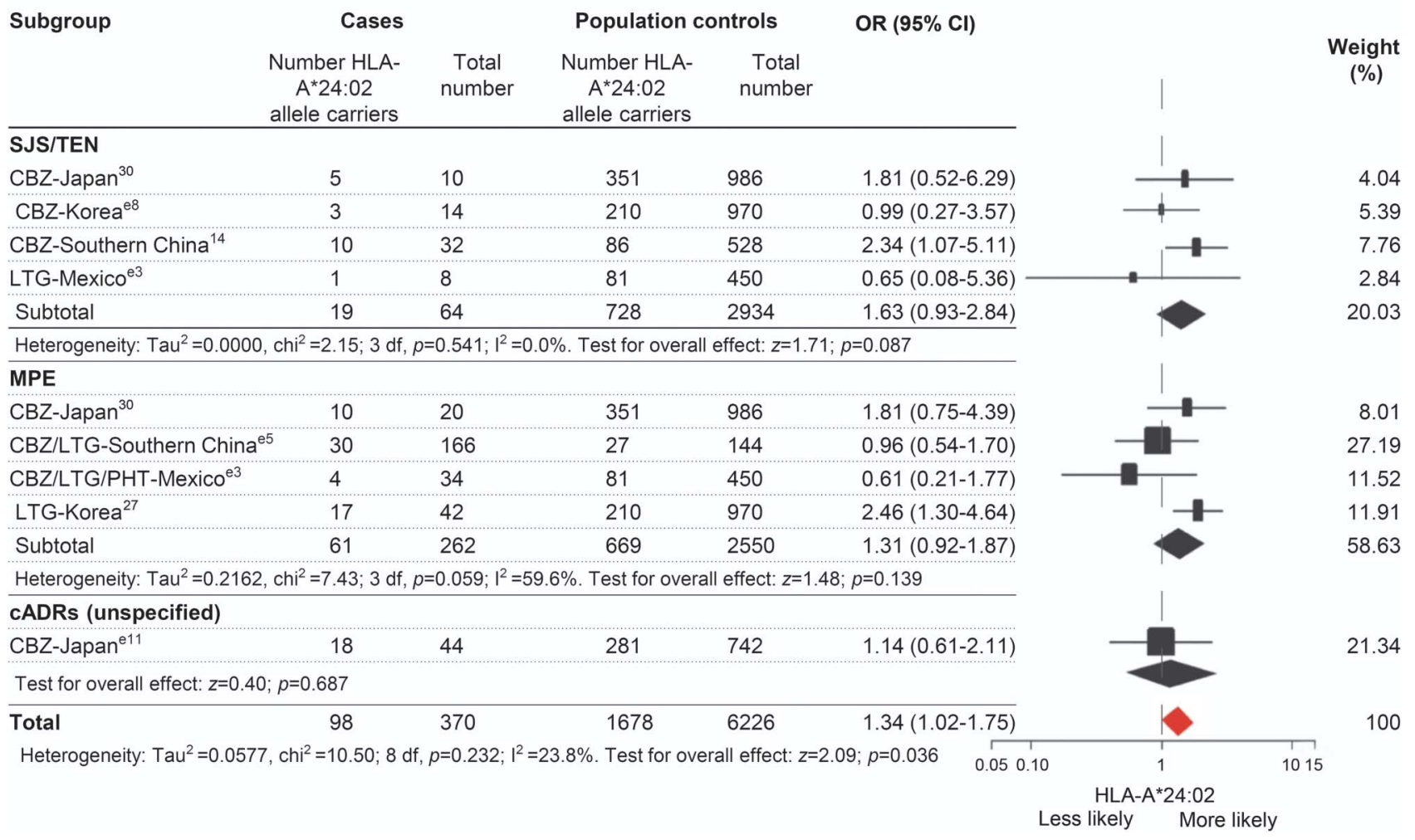


and LTG-induced SJS (22.7\%) in the present cohort (figure 1). Thus, we cannot exclude the possibility that HLA-B*15:02 is a risk allele for PTH- or LTG-induced SJS/TEN. Logistic regression analysis revealed an excess risk from the interaction between HLA-A*24:02 and HLA-B*15:02, suggesting that HLA-A*24:02 functions together with HLA$B * 15: 02$ to contribute to aromatic AED-induced SJS/TEN. HLA class I molecules, including HLAA, HLA-B, and HLA-C, are expressed codominantly on the cell surface. They present peptides to attract killer $\mathrm{T}$ cells (also known as $\mathrm{CD} 8^{+} \mathrm{T}$ cells or cytotoxic $\mathrm{T}$ cells) and are involved in the cytotoxic responses in SJS/TEN. ${ }^{34}$ Thus, it is plausible that more than one HLA locus is associated with drug-induced SJS/TEN and that their effects might be accumulative, as in the case of HLA-B*15:02 and HLA-A*24:02.

In the replication cohort of MPE cases, HLA$A * 24: 02$ showed an association, suggesting that it also has a role in milder cADRs induced by aromatic AEDs. This was supported by the findings of the meta-analysis of data from independent studies in other populations. An association was found between HLA-A*24:02 and MPE or unspecified cADRs in studies using tolerant controls, and the overall association between HLA-A*24:02 and cADRs in data from studies using population controls was statistically significant. Taken together, these data suggested that HLA-A*24:02 is an independent risk factor for severe SJS/TEN, as well as milder MPE. This broad phenotype profile is similar to HLA-A*31:01, which is associated with an increased risk of CBZ-induced SJS, hypersensitivity syndrome, and MPE in white ${ }^{29}$ and Japanese populations. ${ }^{28}$

The meta-analysis demonstrated that HLA$A^{*} 24: 02$ is also a risk factor for aromatic AEDinduced cADRs in populations other than southern Han Chinese. In contrast to HLA-B*15:02, which is specifically prevalent in some populations in southeast Asia, HLA-A*24:02 is prevalent in most of the populations (http://www.allelefrequencies.net). The association between HLA and CBZ-SJS/TEN is generally ethnically specific such as HLA-B*15:02 in populations in south China and southeast Asia, with sensitivities varying from $69.6 \%$ (the present study) to $100 \%,,^{12-17,20}$ and HLA-A*31:01 in white ${ }^{29}$ and Japanese populations. ${ }^{35}$ Notably, the specificity of HLA-B*15:02 for CBZ-SJS/TEN appears to correlate with phenotype severity. ${ }^{36}$ In Taiwanese cases with CBZ-SJS/TEN with $>5 \%$ skin detachment, the HLA-B*15:02 carrier rate was as high as $100 \%$, whereas in more common cases with $<5 \%$ skin detachment, this rate varied from $80.0 \%$ to 87.7\%; HLA-A*31:01 was also demonstrated to be a risk factor for MPE in Taiwanese populations. ${ }^{37}$ These data suggested that ethnic specificity is less significant for the milder phenotype. ${ }^{38}$ In these relatively milder and common cases, an HLA risk factor typically accounts for a portion of the cases such as HLA-A*31: 01 in $42 \%$ of CBZ-induced SJS cases in whites ${ }^{29}$ and $60.7 \%$ of cases in Japanese. ${ }^{28}$ Therefore, the role of other HLA risk factors such as HLA-A*24:02 should be evaluated further.

Our study had some limitations. First, it focused only on HLAs. In addition to HLAs, other genetic and host factors might play a role in aromatic AED-induced cADRs. A recent genome-wide association study of common variants from Taiwan showed that the CYP2C9*3 allele of the drug-metabolizing enzyme might be involved in PHT-induced SJS/ TEN. ${ }^{30}$ The role of rare variants remains to be investigated. Our previous study demonstrated that a history of allergy is a risk factor for oxcarbazepineinduced MPE. ${ }^{39}$ Second, despite recruitment from 8 centers, there were few cases of PHT-induced SJS/ TEN. Third, although the meta-analyses suggested that HLA-A*24:02 is a common risk factor for aromatic AED-induced cADRs in other ethnic populations, further studies in larger cohorts from these populations are needed to verify this association. Indeed, the potential roles of HLA-B*15:11, HLA$B * 15: 01$, and HLA-A*02:01 in aromatic AEDinduced SJS/TEN and the interactions between HLA-A and HLA-B require further verification.

In the present study, positivity for HLA$A * 24: 02$ showed a sensitivity of $36.3 \%$ and a specificity of $84.7 \%$ for aromatic AED-induced SJS/ TEN, which were similar to those for HLA$A^{*} 31: 01$ for CBZ-induced hypersensitivity syndrome observed in northern European populations (37.0\% and $96.1 \%$, respectively). The combination of HLA-A*24:02 and HLA-B*15:02 showed a sensitivity of $72.5 \%$ and a specificity of $69.0 \%$ for aromatic AED-induced SJS/TEN. SJS/TEN is a life-threatening disorder, and HLA-A*24:02 is prevalent; therefore, our findings suggested that

\footnotetext{
Figure 2 legend, continued:

Data were from 14 studies, including 7 that used drug-tolerant controls, 5 that used population controls, and 2 that used both types of controls, including populations from Japan, Korea, Malaysia, Mexico, Norway, and China (e-supplement). Data from tolerant controls (A) and population controls (B) were analyzed separately. The phenotypes of cADRs included SJS/TEN, MPE, and unspecified cADRs, according to the descriptions in the original reports. $I^{2}$ and $\tau^{2}$ and represent measures of heterogeneity. Study weighting (indicated by different sizes of squares) refers to the proportion of participants who were recruited from each study. Horizontal lines represent $95 \% \mathrm{Cls}$; diamonds indicate pooled ORs. AED = antiepileptic drug; cADRs = cutaneous adverse drug reactions; $\mathrm{CBZ}=$ carbamazepine; $\mathrm{Cl}$ = confidence interval; $\mathrm{HLA}$ = human leukocyte antigen; $\mathrm{LTG}$ = lamotrigine; $\mathrm{MPE}=$ maculopapular exanthema; $\mathrm{OR}=$ odds ratio; $\mathrm{PHT}$ = phenytoin; SJS/TEN = Stevens-Johnson syndrome/toxic epidermal necrolysis.
} 
in southern China, screening for HLA-A*24:02 should be considered before aromatic AEDs are prescribed, in addition to screening for HLA-B*15:02 before $\mathrm{CBZ}$ is prescribed. In other populations, further studies in larger cohorts are needed before a similar recommendation can be made.

\section{AUTHOR AFFILIATIONS}

From Institute of Neuroscience and Department of Neurology (Y.-W.S., F.-L.M., J.W., N.H., B.-J.M., X.-R.L., B.-M.L., Y.-Y.K., Y.-H.Y., T.S., W.-P.L.), and Clinical Laboratory (X.-Y.D.), Second Affiliated Hospital of Guangzhou Medical University; Key Laboratory of Neurogenetics and Channelopathies of Guangdong Province (Y.-W.S., F.-L.M., J.W., N.H., B.-J.M., X.-R.L., B.-M.L., Y.-Y.K., Y.-H.Y., T.S., W.-P.L.); Ministry of Education of China (Y.-W.S., F.-L.M., J.W., N.H., B.-J.M., X.-R.L., B.-M.L., Y.-Y.K., Y.-H.Y., T.S., W.-P.L.); Department of Neurology (F.-L.M.), Guangzhou First People's Hospital, Guangzhou Medical University; Department of Neurology (D.Z., F.-Y.H.), West China Hospital, Sichuan University, Chengdu; Epilepsy Center and Department of Neurosurgery (B.Q.), First Affiliated Hospital, Jinan University, Guangzhou; Affiliated Hospital of Xiangnan University (J.W.) Chenzhou; Department of Medicine and Therapeutics (Y.-K.C., P.K.), Chinese University of Hong Kong; Department of Neurology (J.-H.Z. X.-S.H., Y.-M.O.), Guangdong 999 Brain Hospital; Department of Neurology (J.-Q.Z., L.M.-Z.), First Affiliated Hospital, Sun Yat-Set University, Guangzhou; Shanghai Tissuebank Biotechnology Co, Ltd (Z.-Z.Z., J.P.), Shanghai; Guangdong Provincial Key Laboratory of Allergy \& Clinical Immunology (N.H.), Guangzhou; Department of Neurology (Z.-S.L., X.-L.H.), Wuhan Children's Hospital; First People's Hospital of Shunde (Y.-Q.H.), Foshan, China; Division of Neurology (K.-S.L.), Department of Medicine, and Genetics and Molecular Biology Unit (A.H.-P.K., C.-C.N.), Institute of Biological Sciences, University of Malaya, Kuala Lumpur, Malaysia; and Departments of Medicine and Neurology (P.K.), University of Melbourne, Royal Melbourne Hospital, Australia.

\section{AUTHOR CONTRIBUTIONS}

W.P. Liao and P. Kwan conceptualized the study, analyzed and interpreted the data, and drafted and revised the manuscript. W.P. Liao and P. Kwan had full access to all data in the study and take responsibility for the integrity of the data and accuracy of the data analysis. Y.-W. Shi and F.-L. Min analyzed and interpreted the data, drafted and revised the manuscript, and contributed to the statistical analysis. J. Wang and B.J. Mao contributed to the statistical analysis. All authors collected data, revised the manuscript, and contributed to the writing.

\section{ACKNOWLEDGMENT}

We thank Dr. Ley Sander of the UCL Institute of Neurology (London, UK), Dr. C.T. Tan of the University of Malaya Medical Centre (Kuala Lumpur, Malaysia), and Dr. Michael Berkwits of the JAMA Network (Chicago, IL) for their critical reading and helpful suggestions. We are grateful to the He Shanheng Charity Foundation for contributing to the development of our institute.

\section{STUDY FUNDING}

This work was funded by the National Natural Science Foundation of China (grants 81271434, 81301107, 81571273, 81571274, 81501124, and 81601136), the Science and Technology Project of Guangdong Province (grant 2013B051000084), the Department of Education of Guangdong Province (grants 2013CXZDA022, 2013KJCX0156, and 2015KCXTD024), the Foundation for HighLevel Talents in Higher Education of Guangdong (grant 2013167), the Yangcheng Scholar Research Project of Guangzhou Municipal College (grants 12A016S and 12A017G), the Science and Technology Project of Guangzhou (grants 2014J4100069 and 201508020011), the Collaborative Innovation Center for Neurogenetics and Channelopathies, and the Hong Kong Health and Health Services Research (grant 07080381). The funders had no role in study design, data collection, and analysis or in the decision to publish or the preparation of the manuscript.

\section{DISCLOSURE}

Y. Shi, F. Min, D. Zhou, B. Qin, J. Wang, F. Hu, Y. Cheung, J. Zhou, X. Hu, J. Zhou, L. Zhou, Z. Zheng, J. Pan, N. He, Z. Liu, Y. Hou, K. Lim, Y. Ou, A. Khor, C. Ng, B. Mao, X. Liu, B. Li, Y. Kuan, Y. Yi, X. He, X. Deng, and T. Su report no disclosures relevant to the manuscript. P. Kwan has received research grants from the National Health and Medical Research Council of Australia, Australian Research Council, NIH, Hong Kong Research Grants Council, Innovation and Technology Fund, Health and Health Services Research Fund, and Health and Medical Research Fund. He/his institution also received speaker or consultancy fees and/or research grants from Eisai, GlaxoSmithKline, Johnson \& Johnson, Pfizer, and UCB Pharma. W. Liao reports no disclosures relevant to the manuscript. Go to Neurology.org for full disclosures.

Received November 8, 2016. Accepted in final form March 17, 2017.

\section{REFERENCES}

1. Bastuji-Garin S, Rzany B, Stern RS, et al. Clinical classification of cases of toxic epidermal necrolysis, StevensJohnson syndrome, and erythema multiforme. Arch Dermatol 1993;129:92-96.

2. Shear NH, Spielberg SP. Anticonvulsant hypersensitivity syndrome: in vitro assessment of risk. J Clin Invest 1988; 82:1826-1832.

3. Fiszenson-Albala F, Auzerie V, Mahe E, et al. A 6-month prospective survey of cutaneous drug reactions in a hospital setting. Br J Dermatol 2003;149:1018-1022.

4. Chave TA, Mortimer NJ, Sladden MJ, Hall AP, Hutchinson PE. Toxic epidermal necrolysis: current evidence, practical management and future directions. Br J Dermatol 2005;153: 241-253.

5. Roujeau JC, Stern RS. Severe adverse cutaneous reactions to drugs. N Engl J Med 1994;331:1272-1285.

6. Auquier-Dunant A, Mockenhaupt M, Naldi L, Correia O, Schroder W, Roujeau JC; SCAR Study Group, Severe Cutaneous Adverse Reactions. Correlations between clinical patterns and causes of erythema multiforme majus, Stevens-Johnson syndrome, and toxic epidermal necrolysis: results of an international prospective study. Arch Dermatol 2002;138:1019-1024.

7. Roujeau JC, Kelly JP, Naldi L, et al. Medication use and the risk of Stevens-Johnson syndrome or toxic epidermal necrolysis. N Engl J Med 1995;333:1600-1607.

8. Arif H, Buchsbaum R, Weintraub D, et al. Comparison and predictors of rash associated with 15 antiepileptic drugs. Neurology 2007;68:1701-1709.

9. Mallal S, Nolan D, Witt C, et al. Association between presence of HLA-B*5701, HLA-DR7, and HLA-DQ3 and hypersensitivity to HIV-1 reverse-transcriptase inhibitor abacavir. Lancet 2002;359:727-732.

10. Hetherington S, Hughes AR, Mosteller M, et al. Genetic variations in HLA-B region and hypersensitivity reactions to abacavir. Lancet 2002;359:1121-1122.

11. Hung SI, Chung WH, Liou LB, et al. HLA-B*5801 allele as a genetic marker for severe cutaneous adverse reactions caused by allopurinol. Proc Natl Acad Sci USA 2005;102: 4134-4139.

12. Chung WH, Hung SI, Hong HS, et al. Medical genetics: a marker for Stevens-Johnson syndrome. Nature 2004; 428:486.

13. Wang Q, Zhou JQ, Zhou LM, et al. Association between HLA-B*1502 allele and carbamazepine-induced severe 
cutaneous adverse reactions in Han people of southern China mainland. Seizure 2011;20:446-448.

14. Shi YW, Min FL, Qin B, et al. Association between HLA and Stevens-Johnson syndrome induced by carbamazepine in southern Han Chinese: genetic markers besides B*1502? Basic Clin Pharmacol Toxicol 2012;111:58-64.

15. Mehta TY, Prajapati LM, Mittal B, et al. Association of HLA-B*1502 allele and carbamazepine-induced StevensJohnson syndrome among Indians. Indian J Dermatol Venereol Leprol 2009;75:579-582.

16. Tassaneeyakul W, Tiamkao S, Jantararoungtong $\mathrm{T}$, et al. Association between HLA-B*1502 and carbamazepineinduced severe cutaneous adverse drug reactions in a Thai population. Epilepsia 2010;51:926-930.

17. Chang CC, Too CL, Murad S, Hussein SH. Association of HLA-B*1502 allele with carbamazepine-induced toxic epidermal necrolysis and Stevens-Johnson syndrome in the multi-ethnic Malaysian population. Int J Dermatol 2011; 50:221-224.

18. Chen P, Lin JJ, Lu CS, et al. Carbamazepine-induced toxic effects and HLA-B*1502 screening in Taiwan. N Engl J Med 2011;364:1126-1133.

19. Liao WP, Shi YW, Min FL. HLA-B*1502 screening and toxic effects of carbamazepine. N Engl J Med 2011;365: 672-673; author reply 673 .

20. Zhang Y, Wang J, Zhao LM, et al. Strong association between HLA-B*1502 and carbamazepine-induced Stevens-Johnson syndrome and toxic epidermal necrolysis in mainland Han Chinese patients. Eur J Clin Pharmacol 2011;67:885-887.

21. Min FL, Shi YW, Zhou D, et al. HLA-A*2402 as a common genetic risk factor for aromatic antiepileptic drugs induced Stevens-Johnson syndrome and toxic epidermal necrolysis in Han Chinese. Presented at the 10th Asian \& Oceanian Epilepsy Congress; August 8, 2014; Singapore.

22. Roujeau JC. The spectrum of Stevens-Johnson syndrome and toxic epidermal necrolysis: a clinical classification. J Invest Dermatol 1994;102:28S-30S.

23. Trachtenberg E, Vinson M, Hayes E, et al. HLA class I (A, $\mathrm{B}, \mathrm{C})$ and class II (DRB1, DQA1, DQB1, DPB1) alleles and haplotypes in the Han from southern China. Tissue antigens 2007;70:455-463.

24. Hung SI, Chung WH, Jee SH, et al. Genetic susceptibility to carbamazepine-induced cutaneous adverse drug reactions. Pharmacogenet Genomics 2006;16:297-306.

25. Mockenhaupt M, Messenheimer J, Tennis P, Schlingmann J. Risk of Stevens-Johnson syndrome and toxic epidermal necrolysis in new users of antiepileptics. Neurology 2005;64:1134-1138.

26. Bell GS, Neligan A, Sander JW. An unknown quantity: the worldwide prevalence of epilepsy. Epilepsia 2014;55: 958-962.
27. Mockenhaupt M, Viboud C, Dunant A, et al. StevensJohnson syndrome and toxic epidermal necrolysis: assessment of medication risks with emphasis on recently marketed drugs: the EuroSCAR-study. J Invest Dermatol 2008; $128: 35-44$.

28. Ozeki T, Mushiroda T, Yowang A, et al. Genome-wide association study identifies HLA-A*3101 allele as a genetic risk factor for carbamazepine-induced cutaneous adverse drug reactions in Japanese population. Hum Mol Genet 2011;20:1034-1041.

29. McCormack M, Alfirevic A, Bourgeois S, et al. HLAA*3101 and carbamazepine-induced hypersensitivity reactions in Europeans. N Engl J Med 2011;364: 1134-1143.

30. Chung WH, Chang WC, Lee YS, et al. Genetic variants associated with phenytoin-related severe cutaneous adverse reactions. JAMA 2014;312:525-534.

31. Cheung YK, Cheng SH, Chan EJ, Lo SV, Ng MH, Kwan P. HLA-B alleles associated with severe cutaneous reactions to antiepileptic drugs in Han Chinese. Epilepsia 2013;54:1307-1314.

32. Hung SI, Chung WH, Liu ZS, et al. Common risk allele in aromatic antiepileptic-drug induced Stevens-Johnson syndrome and toxic epidermal necrolysis in Han Chinese. Pharmacogenomics 2010;11:349-356.

33. Man CB, Kwan P, Baum L, et al. Association between HLA-B*1502 allele and antiepileptic drug-induced cutaneous reactions in Han Chinese. Epilepsia 2007;48:10151018.

34. Nassif A, Bensussan A, Boumsell L, et al. Toxic epidermal necrolysis: effector cells are drug-specific cytotoxic T cells. J Allergy Clin Immunol 2004;114:1209-1215.

35. Shirzadi M, Thorstensen K, Helde G, Moen T, Brodtkorb E. Do HLA-A markers predict skin-reactions from aromatic antiepileptic drugs in a Norwegian population? A case control study. Epilepsy Res 2015;118:5-9.

36. Ikeda H, Takahashi Y, Yamazaki E, et al. HLA class I markers in Japanese patients with carbamazepineinduced cutaneous adverse reactions. Epilepsia 2010; 51:297-300.

37. Hsiao YH, Hui RC, Wu T, et al. Genotype-phenotype association between HLA and carbamazepine-induced hypersensitivity reactions: strength and clinical correlations. J Dermatol Sci 2014;73:101-109.

38. Moon J, Park HK, Chu K, et al. The HLA-A*2402/Cw*0102 haplotype is associated with lamotrigine-induced maculopapular eruption in the Korean population. Epilepsia 2015;56:e161-e167.

39. He N, Min FL, Shi YW, et al. Cutaneous reactions induced by oxcarbazepine in southern Han Chinese: incidence, features, risk factors and relation to HLA-B alleles. Seizure 2012;21:614-618. 


\section{Neurology}

\section{HLA-A*24:02 as a common risk factor for antiepileptic drug-induced cutaneous adverse reactions}

Yi-Wu Shi, Fu-Li Min, Dong Zhou, et al.

Neurology 2017;88;2183-2191 Published Online before print May 5, 2017

DOI 10.1212/WNL.0000000000004008

This information is current as of May 5, 2017

Neurology ${ }^{\circledR}$ is the official journal of the American Academy of Neurology. Published continuously since 1951, it is now a weekly with 48 issues per year. Copyright Copyright (C) 2017 The Author(s). Published by Wolters Kluwer Health, Inc. on behalf of the American Academy of Neurology. All rights reserved. Print ISSN: 0028-3878. Online ISSN: 1526-632X.

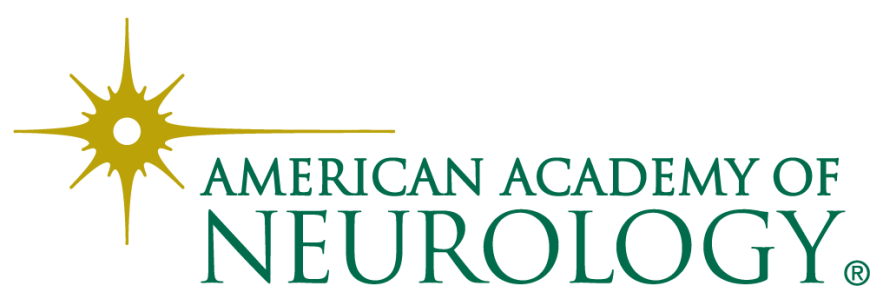




\section{Updated Information \& Services}

\section{Supplementary Material}

References

Citations

Subspecialty Collections

Permissions \& Licensing

\section{Reprints}

including high resolution figures, can be found at: http://n.neurology.org/content/88/23/2183.full

Supplementary material can be found at: http://n.neurology.org/content/suppl/2017/05/05/WNL.0000000000004 008.DC1

This article cites 38 articles, 3 of which you can access for free at: http://n.neurology.org/content/88/23/2183.full\#ref-list-1

This article has been cited by 1 HighWire-hosted articles: http://n.neurology.org/content/88/23/2183.full\#\#otherarticles

This article, along with others on similar topics, appears in the following collection(s):

\section{All Genetics}

http://n.neurology.org/cgi/collection/all_genetics

All Toxicology

http://n.neurology.org/cgi/collection/all_toxicology

Antiepileptic drugs

http://n.neurology.org/cgi/collection/antiepileptic_drugs

Case control studies

http://n.neurology.org/cgi/collection/case_control_studies

Patient safety

http://n.neurology.org/cgi/collection/patient_safety

Information about reproducing this article in parts (figures,tables) or in its entirety can be found online at:

http://www.neurology.org/about/about_the_journal\#permissions

Information about ordering reprints can be found online:

http://n.neurology.org/subscribers/advertise

Neurology ${ }^{\circledR}$ is the official journal of the American Academy of Neurology. Published continuously since 1951, it is now a weekly with 48 issues per year. Copyright Copyright ( 2017 The Author(s). Published by Wolters Kluwer Health, Inc. on behalf of the American Academy of Neurology. All rights reserved. Print ISSN: 0028-3878. Online ISSN: 1526-632X.

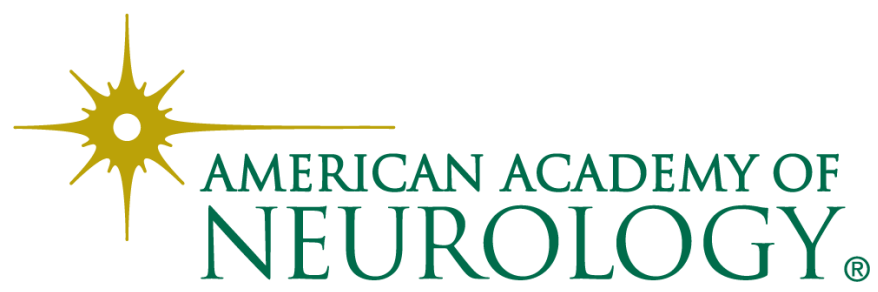

